\title{
Comparative Study Between the efficacy of Long- Pulsed Neodymium- YAG Laser and Fractional Co2 Laser in the Treatment of Striae Distensae
}

\author{
Alyaa Farouk Hendawy ${ }^{1 *(\infty}$, Dalia Gamal Aly ${ }^{\circledR}$, Hisham Aly Shokeir ${ }^{2}$, Nevien Ahmed Samy ${ }^{2}$ \\ 'Department of Dermatology and Venereology, National Research Centre, Giza, Egypt \\ ${ }^{2}$ Department of Medical Applications of Laser in Dermatology, NILES, Cairo, Egypt
}

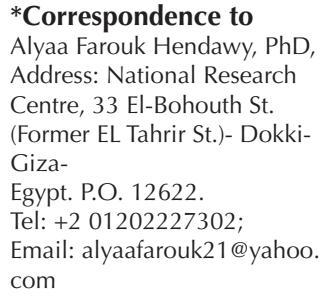

*Correspondence to

Alyaa Farouk Hendawy, PhD Address: National Research Centre, 33 El-Bohouth St.

(Former EL Tahrir St.)- DokkiGiza-

Egypt. P.O. 12622

Tel: +2 01202227302

Email: alyaafarouk21@yahoo. com

Received: March 19, 2021 Accepted: July 7, 2021 Published online October 9 2021

\begin{abstract}
Introduction: Stretch marks, or striae distensae (SD), are the lesions of the dermis caused by its linear atrophy at the sites of stretching. They occur in more than $70 \%$ of pregnant women and adolescents as a result of cutaneous stretching. This study aimed at evaluating and comparing the clinical and histological efficacy of the fractional $\mathrm{CO}_{2}$ laser versus the long pulsed (LP) $\mathrm{Nd}$ : YAG laser in the treatment of striae alba.

Methods: Thirty female subjects having bilateral symmetrical stretch marks were managed by the LP $\mathrm{Nd}$ : YAG laser on the right side and the fractional $\mathrm{CO}_{2}$ laser on the left side. The laser treatment course consisted of 3 sessions with an interval of 3 weeks. The patient satisfaction score and the Global Aesthetic Improvement Scale (GAIS) were employed in the study in order to assess the improvement 3 months after therapy. Punch biopsies measuring $4 \mathrm{~mm}$ were extracted from one lesion on each side at baseline and after the last treatment session by 3 months to evaluate the thickness of both collagen and epidermis

Results: Clinical improvement was more significant in the lesions treated by the LP Nd:YAG laser than those treated with the fractional $\mathrm{CO}_{2}$ laser. The patient satisfaction score and the GAIS were higher with a statistically significant value in the side treated by the LP Nd:YAG laser. Collagen and epidermal thickness increased more in the LP Nd: YAG laser-treated lesions in comparison to their thickness in the fractional $\mathrm{CO}_{2}$ laser-managed lesions, but this difference did not have a statistical significance.

Conclusion: The clinical efficacy of the LP Nd-YAG laser exceeded that of the fractional $\mathrm{CO}_{2}$ laser in the treatment of striae alba without severe side effects in spite of the insignificant histological difference between the two lasers.

Keywords: Stretch marks; Striae alba; Long pulsed (LP) Nd-YAG Laser; Fractional $\mathrm{CO}_{2}$ laser.
\end{abstract}

\section{Introduction}

Striae distensae (SD), commonly named stretch marks, are a type of dermal scarring that arise on the skin as erythematous, violaceous, or hypopigmented linear patterns. ${ }^{1}$ Stretch marks associated with pregnancy are called 'striae gravidarum' and occur in more than $70 \%$ of pregnant females. The pathogenesis of SD is not well identified and is perhaps multifactorial, but it is clearly due to changes in the structures that give the skin its strength and elasticity. ${ }^{2}$

Physiological states like fast weight gain, adrenocortical excess, and pregnancy, and pathological states such as longterm use of either systemic or topical steroids, Cushing and Marfan syndromes, as well as genetic alterations in connective tissue with reduced expression of fibronectin and collagen genes in involved tissue, might altogether add to the occurrence of SD. ${ }^{3}$ Although SD do not result in any medical problems, they cause psychological stress for many women. ${ }^{4}$

Though numerous options have been tried to treat and improve the clinical appearance of SD, most have yielded suboptimal results. ${ }^{5}$ Such treatments include topical retinoids, microneedling, chemical peeling, radiofrequency, platelet-rich plasma, and ablative and nonablative lasers. ${ }^{5}$ Lasers used for treating stretch marks include diode laser, 308-nm excimer laser, 1064-nm neodymium-doped yttrium aluminum garnet (Nd: YAG) laser, 585-nm pulse dye laser and ablative fractional $\mathrm{CO}_{2}$ resurfacing laser. ${ }^{6}$

The fractional ablative $\mathrm{CO}_{2}$ laser was used primarily for treating surgical and acne scars because of its capability of triggering elastin regeneration as well as collagen remodeling. Furthermore, it has been reported that the fractional $\mathrm{CO}_{2}$ laser induces new collagen formation to 
a superior degree than the nonablative lasers. ${ }^{7}$ Several studies in the literature have reported its safety and efficacy in treating SD given the fact that SD is considered a type of a dermal depressed atrophic scar. ${ }^{8}$

The 1064-nm long pulsed (LP) Nd: YAG laser is well recognized for treating vascular lesions and has also exhibited the capability to stimulate the formation of dermal collagen when used to treat facial rhytides. ${ }^{9}$ The combination of collagen induction and vascular selectivity has confirmed to be beneficial in treating immature striae rubra in patients with skin phototypes II-IV. ${ }^{10}$

To our knowledge, no previous research works have compared the effects of the fractional $\mathrm{CO}_{2}$ laser and the LP Nd: YAG laser on the treatment of stretch marks. Thus, the aim of the present study was to assess and compare the clinical and histological effectiveness of the fractional $\mathrm{CO}_{2}$ laser and the LP Nd: YAG laser in treating striae alba.

\section{Patients and Methods}

This study was a prospective intra-patient comparative study. The screening of 70 women having striae alba and Fitzpatrick skin type III-V was performed, leaving only 46 females who fulfilled the inclusion criteria. Two female candidates were dropped after the first treatment session, 13 candidates were dropped after the second treatment session, and only one candidate was dropped after the third treatment session. As a result, in this study, we had 30 female patients who completed their treatment sessions. The sites of SD included the flanks, breasts and thighs. The duration of SD lesions varied from 1 to 20 years. The age of the patients was in the range of 18-35 years. Exclusion criteria included patients with striae rubra, pregnant and lactating women, patients treated by topical or systemic steroids, retinoids, oral contraceptive pills, hormone replacement therapy, anticoagulants and immunomodulators. In addition, females having systemic disorders such as diabetes, hypertension, Cushing syndrome, goitre, asthma, connective tissue disorders, bleeding or coagulation disorders and immunocompromised states were not included in the study. Also, patients having liability to develop keloids or post-inflammatory hyperpigmentation (PIH) were excluded from the study. The random recruitment of the patients was done at the dermatology clinic, National Research Centre (NRC), Giza, Egypt.

Stretch marks were assessed to detect their sites and their types. The assessment of the extent of the lesions was established by the measurement of both width and length of SD. The evaluation of the treated SD was done before the treatment, at the beginning of each session and 3 months after the third session.

\section{Management of Striae With Fractional $\mathrm{CO}_{2}$ and $\mathrm{Nd}$ :YAG Lasers}

Two bilateral symmetrical striae alba were chosen in every patient (left and right). The Fractional $\mathrm{CO}_{2}$ laser was applied to $\mathrm{SD}$ on the left side using the Pixel $\mathrm{CO}_{2}$ resurfacing system, China, at the energy of $60 \mathrm{~mJ}$, the scanning area of $5 \mathrm{~mm} \times 5 \mathrm{~mm}$ and the pulse duration of $2 \mathrm{~ms}$. One pass of fractional $\mathrm{CO}_{2}$ was applied to the SD lesions. On the other hand, the LP Nd: YAG laser was applied to striae on the right side using the Fotona XP Dynamis laser machine, Slovenia. To determine the suitable parameters of the LP Nd:YAG laser in order to be used in this study, we tested several parameters on volunteers before recruiting our patients or starting this research. On using low parameters, the response was not satisfactory for both the patients and the physician. On the other hand, using high parameters resulted in pain and a higher incidence of side effects, especially erythema and edema that lasted for several days, followed by hyperpigmentation as the case in the Egyptian patients' skin type. Accordingly, the parameters used were $80 \mathrm{~J} /$ $\mathrm{cm}^{2}$ fluence, 4-mm spot size and $15-\mathrm{ms}$ pulse duration, which are relatively near to those used by Elsaie et al. ${ }^{11}$ The 4-mm spot size was enough to completely cover the width of all lesions included in the study. Two laser passes of LP Nd:YAG were applied to the SD lesions. Three laser sessions were done in all patients with a 3-week interval. Cooling by cold air was done during the session in order to diminish any discomfort. In addition, goggles and wet gauze pads were used to protect the eyes. All subjects were requested to mention any side effects during the course of laser treatment, such as redness, swelling, itching, pain, pigmentation or crustation. An antibiotic was applied topically 3 times daily for 3 consecutive days after the treatment session.

\section{Histological Evaluation of Striae Alba}

Punch biopsies measuring $4 \mathrm{~mm}$ were extracted from SD lesions from seven candidates chosen randomly. In each candidate, two biopsies were taken at baseline (one from the side that was treated by the fractional $\mathrm{CO}_{2}$ laser and one from the side that was treated by the LP Nd:YAG laser). Another two biopsies were extracted from the same lesions after the last session by 3 months.

All biopsies first emerged in 10\% buffered formalin, and then they were processed into paraffin blocks and finally cut into paraffin sections. All sections were stained with Hematoxylin and eosin (H\&E) for evaluating the lesions histologically and Masson trichrome (MT) stain for examining collagen fibers. A blinded dermatopathologist assessed all skin biopsies histologically at baseline and after the treatment by the Fractional $\mathrm{CO}_{2}$ and LP Nd: YAG lasers.

\section{Examination of Collagen Fibers}

The morphometric evaluation of collagen fibers was carried out at the Pathology Department, NRC, using the Leica Qwin 500 Image Analyzer (LEICA Imaging Systems 
Ltd, Cambridge, England) which is composed of the Leica DM-LB microscope and the JVC color video camera attached to a computer system Leica Q 500IW. Random quantitative morphometric analysis was performed in 5 high-power fields.

Quantitative morphometric analysis of all slides stained by Masson Trichrome was done using $\times 10$ objective lens. The color detect software was employed in order to determine the collagen amount. The recording of the mean area of the collagen fibers in Masson trichrome stained sections was carried out by a binary image on a real-time image from the microscope that was visualized on the video monitor. Statistical analysis of the mean area percent given by the image analyzer was performed, and the ANOVA test was used to assess significant variations in the findings between the groups.

\section{Photographic evaluation}

Photos of SD were taken before starting the treatment, at the beginning of each session and 3 months after the last treatment session using the same camera (Panasonic L28 camera, 8.1 megapixel, China). The photographic assessment was carried out by two blinded dermatology specialists in accordance with the width and length of SD at baseline and after the laser treatment course.

\section{Global Aesthetic Improvement Scale}

The Global Aesthetic Improvement Scale (GAIS) was employed in order to assess the degree of the improvement of SD 3 months after the third treatment session, in which $1=$ very much improved, $2=$ much improved, $3=$ Improved, $4=$ No change and $5=$ Worse. $^{11}$

\section{Patient Satisfaction Score}

The patients uttered their satisfaction as follows: 1 = very satisfied, 2 = satisfied, $3=$ neither satisfied nor unsatisfied, $4=$ unsatisfied and $5=$ very unsatisfied. ${ }^{11}$

\section{Statistical Investigation}

Analysis of the data was done by IBM $^{\star}$ SPSS $^{\star}$ (SPSS Inc., IBM Corporation, NY, USA) Statistics version 26 for Windows. Categorical records were explored as percentages (\%) and frequencies (n), and they underwent analysis by Fisher exact test. The quantitative data were presented for normality using the Shapiro-Wilk and Kolmogorov-Smirnov tests. Analysis of parametric data was done by the paired-samples $t$ test, whereas analysis of nonparametric data was done by the Wilcoxon signed rank test. For all tests, the significance value was set at $P$ $\leq 0.05$.

\section{Results}

30 women whose age ranged from 19 to 35 years with mean \pm standard deviation (SD) of $28.3 \pm 4.8$ years were included in the current study, and they had striae alba. The lesions duration was in the range of 4-20 years and most of the patients (56.7\%) had their SD for 6-10 years. The reasons for stretch marks were either increasing weight in $27(90.0 \%)$ patients or pregnancy in $3(10 \%)$ patients. The sites of the lesions varied: 8 (26.7\%) female candidates had SD on the knees, $5(16.7 \%)$ on the arms, $5(16.7 \%)$ on the legs, $3(10.0 \%)$ on the buttocks, $2(6.7 \%)$ on the shoulders, $2(6.7 \%)$ on the thighs, $2(6.7 \%)$ on the back, $2(6.7 \%)$ on the flanks, and only $1(3.3 \%)$ on the breast.

The majority of the subjects $(80.0 \%)$ included in the study had a family history of stretch marks. Nineteen (63.3\%) subjects had skin type III, 8 (26.7\%) subjects had skin type IV and $3(10.0 \%)$ subjects had skin type V. The BMI index for all patients varied from 22 to 30 with mean $\pm \mathrm{SD}$ of $25.7 \pm 2.5$.

\section{Length and Width of Striae Alba Before and After the Treatment}

Fractional $\mathrm{CO}_{2}$ Laser

The length and width of lesions treated with the fractional $\mathrm{CO}_{2}$ laser improved significantly after a treatment course of 3 laser sessions. The length of the lesions ranged from 1 to $4 \mathrm{~mm}$ before the treatment and reached 0 to $3 \mathrm{~mm}$ after the treatment with mean \pm SD of $2.0 \pm 1.0$ and $1.2 \pm 1.0$ $\mathrm{mm}$ respectively $(P=0.011)$. However, the width of the lesions before the treatment ranged from 1 to $3 \mathrm{~mm}$ and diminished to 0 to $3 \mathrm{~mm}$ after the treatment with mean $\pm \mathrm{SD}$ of $1.7 \pm 0.7$ and $1.0 \pm 0.8 \mathrm{~mm}$ respectively $(P=0.009)$ (Table 1, Figures 1 and 2).

\section{Long Pulsed Nd: YAG Laser}

The length and width of the stretch marks also significantly improved after a treatment course of 3 laser sessions using the LP Nd:YAG laser as the length of SD was in the range of 1 to $4 \mathrm{~mm}$ before the treatment and was $0-3 \mathrm{~mm}$ after the treatment with mean \pm SD of $2.0 \pm 0.9 \mathrm{~mm}$ and $0.6 \pm 0.8$ mm respectively $(P=0.011)$. However, the width of SD lesions before the treatment varied from 1 to $3 \mathrm{~mm}$ and reached $0-2 \mathrm{~mm}$ after the treatment with mean \pm SD $1.4 \pm 0.5 \mathrm{~mm}$ and $0.5 \pm 0.5 \mathrm{~mm}$ respectively $(P=0.009)$ (Table 1, Figures 1 and 2).

The comparison of the two laser treatments revealed that the lesions treated by the LP Nd:YAG laser showed a more significant improvement regarding the length and width of SD than the Fractional $\mathrm{CO}_{2}$ laser-treated lesions $(P=0.009$ and $P=0.011$ versus $P=0.147$ and $P=1$, respectively) (Table 2, Figures 1 and 2).

\section{Global Aesthetic Improvement Scale Score and Patient Satisfaction Score}

The GAIS values after the treatment were significantly higher in the case of LP Nd: YAG laser treatment compared to fractional $\mathrm{CO}_{2}$ laser treatment, as 7 (23.3\%) lesions demonstrated very much improvement and 12 (40\%) lesions demonstrated much improvement versus 16 
(53.3\%) and 8 (26.7\%) lesions that showed improvement and no change respectively after fractional $\mathrm{CO}_{2}$ laser treatment $(P<0.001)$. Moreover, 3 months after therapy, the cases expressed higher satisfaction with LP Nd: YAG laser management than fractional $\mathrm{Co} 2$ laser management $(P<0.001)$.

\section{Examination of Adverse Events}

The subjects had more adverse events with the fractional $\mathrm{CO}_{2}$ laser in comparison to the LP Nd-YAG laser $(P<0.001)$. Twenty-five $(83.3 \%)$ cases had erythema in fractional $\mathrm{CO}_{2}$ laser-managed areas compared to $4(13.3 \%)$ cases after the therapy by the LP ND-YAG laser. In addition, permanent $\mathrm{PIH}$ occurred more with the fractional $\mathrm{CO}_{2}$ laser, as it occurred in $5(16.7 \%)$ cases compared to $0 \%$ of the cases after the LP Nd-YAG laser treatment. No patients complained of infections, burns or scars.

\section{Histological Examination}

Thickness of Epidermis

There was an increase in the thickness of the epidermis after the two laser treatments, LP Nd: YAg laser and fractional $\mathrm{CO} 2$ laser treatments, but without any statistically significant value (mean \pm SD for the LP Nd: YAG laser at baseline: $130.0 \pm 22.4 \mu \mathrm{m}$ versus after the therapy: $183.8 \pm 10.4 \mu \mathrm{m}, P=0.182$; mean \pm SD for the fractional $\mathrm{CO}_{2}$ laser at baseline: $128.2 \pm 19.8 \mu \mathrm{m}$ and after the therapy: $187.5 \pm 15.9 \mu \mathrm{m}, P=0.1$ ) (Table 3, Figures 3 and 4).

\section{Thickness of Collagen}

After the treatment, there was an increase in the mean area percent of collagen with both LP Nd: YAg laser and fractional $\mathrm{CO}_{2}$ laser managements; however, it did not have a statistical significance (mean \pm SD before the

Table 1: Length and Width of Striae Distensae Treated by the Fractional $\mathrm{CO}_{2}$ Laser and the LP Nd:YAG Laser Before and After the Treatment

\begin{tabular}{|c|c|c|c|c|}
\hline Laser & Parameter & Before Treatment & After Treatment & $P$ Value \\
\hline \multirow{4}{*}{ Fractional $\mathrm{CO}_{2}$} & $\begin{array}{c}\text { Width } \\
\text { Mean } \pm \text { SD }\end{array}$ & $1.7 \pm 0.7$ & $1.0 \pm 0.8$ & \multirow[t]{2}{*}{$<0.001^{*}$} \\
\hline & Median [Range] & $2.00[1.00-3.00]$ & $0.00[0.00-3.00]$ & \\
\hline & Length & $2.0 \pm 1.0$ & $1.2 \pm 1.0$ & \multirow{2}{*}{$<0.001^{*}$} \\
\hline & Median [Range] & $2.00[1.00-4.00]$ & $1.00[0.00-3.00]$ & \\
\hline \multirow{4}{*}{ Nd:YAG } & $\begin{array}{c}\text { Width } \\
\text { Mean } \pm \text { SD }\end{array}$ & $1.4 \pm 0.5$ & $0.5 \pm 0.5$ & \multirow[t]{2}{*}{$<0.001^{*}$} \\
\hline & Median [Range] & $1.00[1.00-3.00]$ & $0.00[0.00-2.00]$ & \\
\hline & Length & $2.0 \pm 0.9$ & $0.6 \pm 0.8$ & \multirow{2}{*}{$<0.001^{*}$} \\
\hline & Median [Range] & $2.00[1.00-4.00]$ & $0.25[0.00-3.00]$ & \\
\hline
\end{tabular}

Table 2: Comparison Between the Effect of the Fractional $\mathrm{CO}_{2}$ Laser and the LP Nd: YAG Laser on the Width and Length of Striae Distensae

\begin{tabular}{|c|c|c|c|c|}
\hline Time & Parameter & Fractional $\mathrm{CO}_{2}$ Laser & Nd: YAG Laser & $P$ value \\
\hline \multirow{2}{*}{ Before treatment } & Width & $1.73 \pm 0.74$ & $1.47 \pm 0.57$ & $0.147^{\text {ns }}$ \\
\hline & Length & $2.07 \pm 0.90$ & $2.07 \pm 1.01$ & $1^{\mathrm{ns}}$ \\
\hline \multirow{2}{*}{ After treatment } & Width & $1.00 \pm 0.83$ & $0.50 \pm 0.57$ & $0.009 *$ \\
\hline & Length & $1.25 \pm 1.06$ & $0.65 \pm 0.83$ & $0.011 *$ \\
\hline
\end{tabular}

* Significant $(P$ value $\leq 0.05)$; ns: non-significant $(P$ value $>0.05)$.

Table 3. Epidermal and Collagen Thickness in Striae Distensae Treated by Each of the Fractional CO2 Laser and the Nd:YAG Laser Before and After the Treatment.

\begin{tabular}{lccc}
\hline Laser & Parameter & Before Treatment & After Treatment \\
\hline & Epidermis & $128.2 \pm 19.8$ & $187.5 \pm 15.9$ \\
Mractional CO 2 & Median [Range] & $129.4[77.8-177.5]$ & $190.0[160.1-210.2]$ \\
& Collagen & $1.2 \pm 0.8$ & $4.2 \pm 1.0$ \\
& Median [Range] & $1.7[0.1-1.7]$ & $2.7[2.2-7.6]$ \\
& Epidermis & $130.0 \pm 22.4$ & $183.8 \pm 10.4$ \\
Md: YAG & Cedian [Range] & $129.6[87.9-172.7]$ & $188.4[171.9-191.2]$ \\
& Collagen & $0.8 \pm 0.6$ & $3.5 \pm 1.4$ \\
\hline
\end{tabular}

ns: non-significant $(P$ value $>0.05)$. 

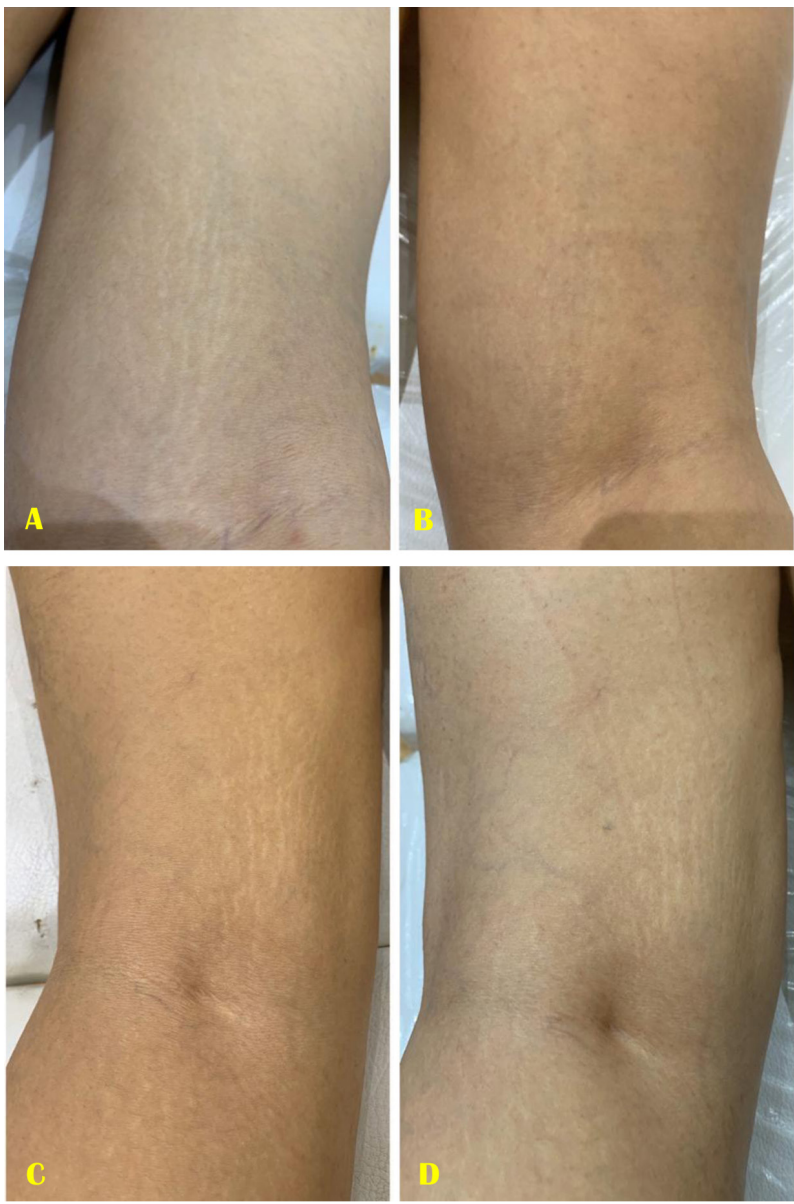

Figure 1. (A) The right thigh of a 26-year-old female patient before Nd: YAG treatment, (B) After three Nd: YAG treatment sessions, (C) The left thigh of the same patient before fractional $\mathrm{CO}_{2}$ laser treatment (D) After three fractional $\mathrm{CO}_{2}$ laser sessions.

treatment for the LP Nd: YAG laser: $0.8 \pm 0.6 \mu \mathrm{m}$ and after the therapy: $3.5 \pm 1.4 \mu \mathrm{m}, P=0.179$; mean \pm SD before the treatment for the fractional $\mathrm{CO}_{2}$ laser: $1.2 \pm 0.8 \mu \mathrm{m}$ and after the therapy: $4.2 \pm 1.0 \mu \mathrm{m}, P=0.116 \mathrm{~ns}$ ).

On comparing the two treatment options as regards the thickness of both epidermis and collagen, there was a more increase in their thickness in lesions managed by the LP Nd: YAG laser than those treated by the fractional $\mathrm{CO}_{2}$ laser. But the difference was not statistically significant $(P=0.8$ for collagen thickness and 0.9 for epidermal thickness).

\section{Discussion}

The aim of the present study was the clinical and histopathological evaluation and comparison of the efficacy of the LP Nd: YAG laser and the fractional $\mathrm{CO}_{2}$ laser in treating stretch marks. Thus, 30 women complaining of SD with an age varying from 19 to 35 years were included in the study.

In the current work, all lesions treated with either the LP Nd: YAG laser or the fractional $\mathrm{CO}_{2}$ laser showed clinical improvement as the length and width of stretch marks diminished after the therapy, but the histological improvement in both treated lesions did not reach a significant level after the treatment. Moreover, the comparison of both laser options demonstrated more statistically significant clinical improvement in SD treated with the LP Nd: YAG laser than those managed by the fractional $\mathrm{CO}_{2}$ laser. In addition, the patient satisfaction score and the GAIS score were significantly higher in SD managed by the LP Nd: YAG laser.

The results of the current work are in agreement with many reports in the literature that confirmed the effectiveness of fractional photothermolysis in the treatment of SD. Yang et $\mathrm{al}^{12}$ reported the efficacy of the $\mathrm{CO} 2$ laser in treating stretch marks. In addition, Lee et $\mathrm{al}^{13}$ applied the ablative fractional $\mathrm{CO}_{2}$ laser to $\mathrm{SD}$ in 27 cases. The lesions were managed in one session using a deep FX mode at the energy of $10 \mathrm{~mJ}$ and a density of $2(10 \%$ coverage). The study concluded that the fractional $\mathrm{CO}_{2}$ laser could be efficient in the treatment of stretch marks. Also, Cho et $\mathrm{al}^{14}$ treated SD with the fractional $\mathrm{CO}_{2}$ laser and showed a significant improvement of lesions after therapy.

The conclusion of the study done by Elsaie et $\mathrm{a}^{11}$ is in agreement with the conclusion of this study regarding the safety and effectiveness of the LP Nd: YAG laser in the treatment of stretch marks. Additionally, Zaleski-Larsen et $\mathrm{al}^{15}$ treated 20 patients having striae alba with the LP

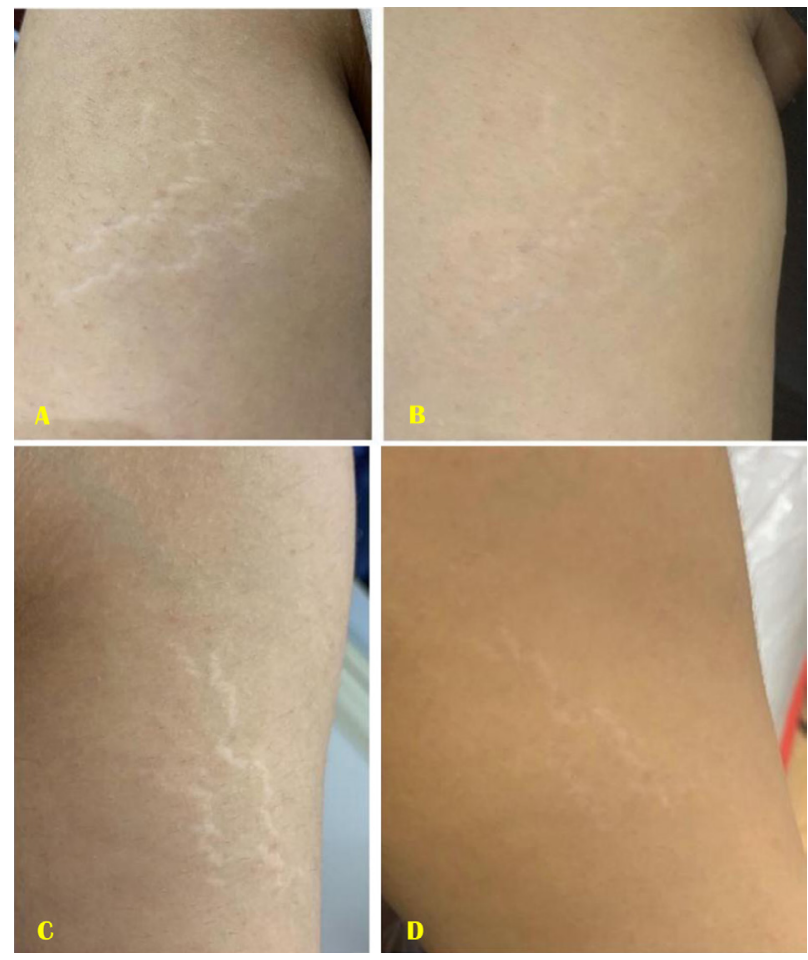

Figure 2. (A) The right arm of a 29-year-old female patient before $\mathrm{Nd}$ : YAG treatment, (B) After three Nd: YAG treatment sessions, (C) The left arm of the same patient before fractional $\mathrm{CO}_{2}$ laser treatment (D) After three fractional $\mathrm{CO}_{2}$ laser sessions. 

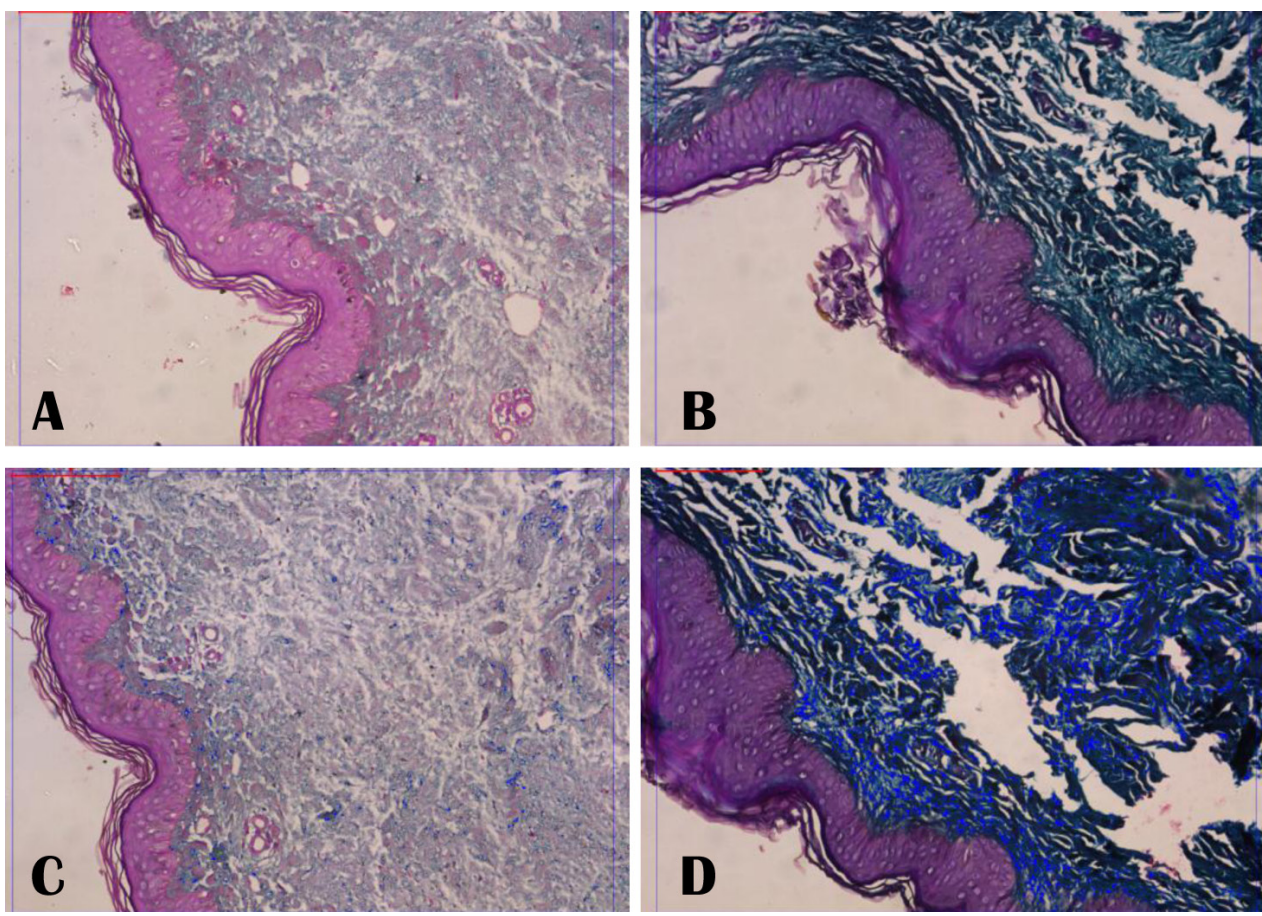

Figure 3. (A) Skin biopsy of untreated striae on thighs and (B) biopsy of the same site of striae treated by the long-pulsed Nd: YAG laser 3 months after the last session. Note the increased epidermal thickness and the thickness of dermal collagen (hematoxylin and eosin, $\times 40$ ) (C) Skin biopsy of untreated striae on thighs and (D) biopsy of the same site of striae treated by the long-pulsed ND YAG laser at the end of the study (3 months after the last session). Note the increased collagen density (Masson trichrome).
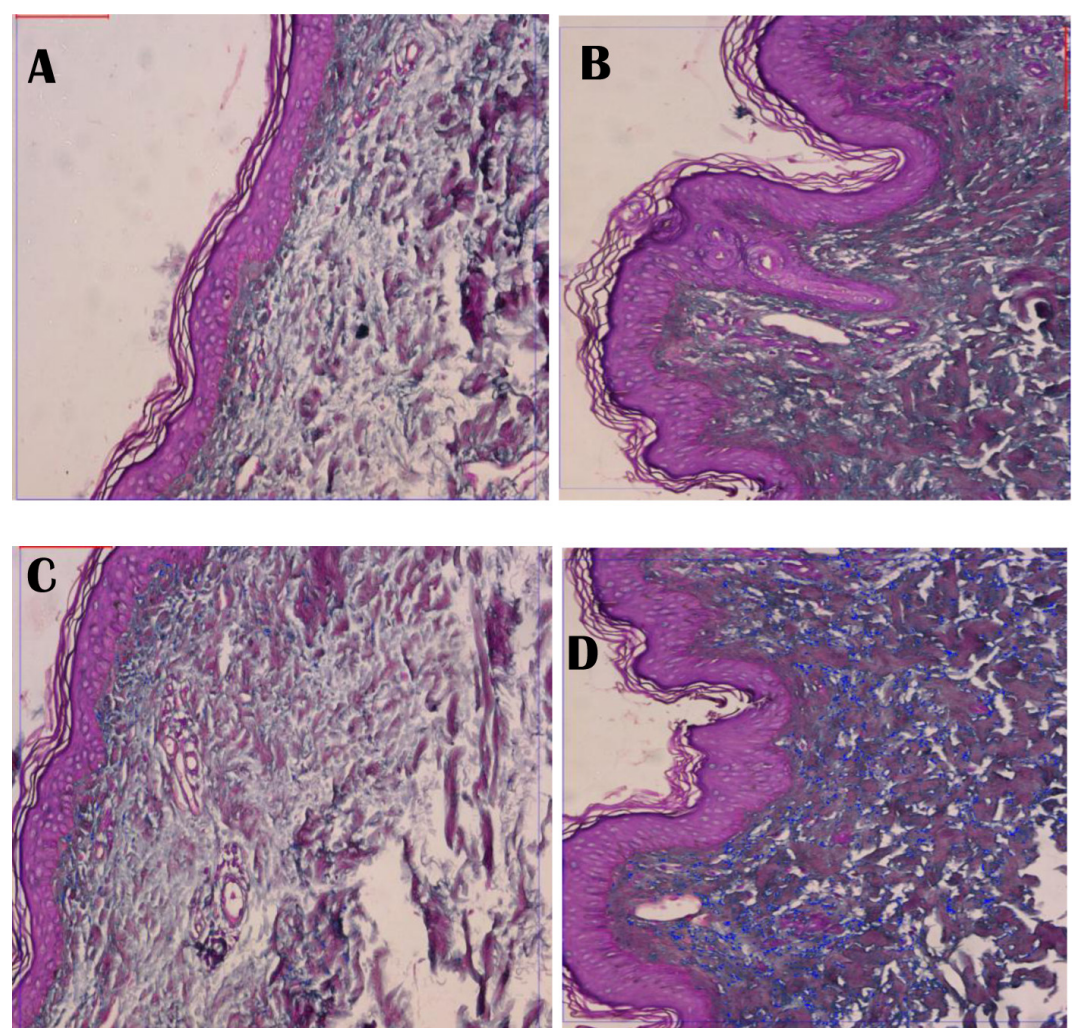

Figure 4. (A) Skin biopsy of untreated striae on thighs and (B) biopsy of the same site of striae treated by the fractional $\mathrm{CO}_{2}$ laser 3 months after the last session. Note the increased epidermal thickness and the thickness of dermal collagen (hematoxylin and eosin, $\times 40)(C)$ Skin biopsy of untreated striae on thighs and (D) biopsy of the same site of striae treated by the fractional $\mathrm{CO}_{2}$ laser at the end of the study $(3$ months after the last session). Note the increased collagen density (Masson trichrome). 
Nd: YAG laser, and there was an improvement in the lesions.

In the present work, although a significant clinical improvement was observed in lesions treated by the two laser types, there was no significant histological improvement regarding both the collagen and epidermal thickness after the last laser session by 3 months. The epidermal and collagen thicknesses were higher in SD managed by the LP ND: YAG laser than those managed by the fractional $\mathrm{CO}_{2}$ laser, but the difference between the two lasers was statistically insignificant.

Disagreeing with this study conclusion was the research work carried out by Güngör et $\mathrm{al}^{16}$ who compared the 2940 nm Er: YAG laser and the LP Nd: YAG laser in 22 candidates suffering from SD. The SD on the two sides of the body were randomly subjected to the two types of lasers, and 3 laser sessions were performed in all cases at one-week intervals. Striae rubra showed a moderate clinical improvement, whereas striae alba showed a poor clinical improvement to therapy on both sides. Histological improvement was demonstrated after the therapy on both sides despite the poor clinical response noted in the two sides.

The previous result can be explained in accordance with Aust et $\mathrm{al},{ }^{17}$ who reported that the deposition of collagen and elastic fibers reached its significant level at 6 months after treatment, and the thickness of the epidermis increased significantly 1 year after treatment, which was not done in this study as there was only a 3-month followup period which is considered short.

The LP Nd: YAG laser has a strong affinity with vascular structures and at the same time causes an increase in dermal collagen, leading to the improvement of immature SD. As the dilated venules and perivascular infiltrate of lymphocytes present in SD lesions are the desirable targets of the Nd: YAG laser due to their content of oxyhemoglobin, the LP Nd: YAG laser causes deposition of a good energy packet in the dermal tissue. ${ }^{18}$

When the LP Nd: YAG laser is absorbed by the dermal vessels, an increase in the temperature around these vessels is produced, causing thermal damage sufficient to trigger wound healing and collagen remodeling. ${ }^{5}$ Moreover, this laser stimulates the formation of new collagen fibers augmenting the improvement of stretch marks. ${ }^{19}$

In the current work, adverse events were noticed more with the fractional $\mathrm{CO}_{2}$ laser than the LP Nd: YAG laser as $83.3 \%$ of the cases complained of erythema in the fractional $\mathrm{CO}_{2}$ laser-treated lesions in comparison to $13.3 \%$ of the cases after the management with the LP Nd: YAG laser. Moreover, permanent PIH was noticed more in the fractional $\mathrm{CO}_{2}$ laser-treated areas in $16.7 \%$ of the patients compared to $0 \%$ of the patients after the therapy with the LP Nd: YAG laser. No patients suffered from infections, burns or scars.

The research work performed by Güngör et $\mathrm{al}^{16}$ was in line with our findings as it showed that long-term mild redness and PIH were observed in 6 out of 20 candidates managed by the Er: YAG laser, while there were no side effects on the LP Nd: YAG laser managed areas.

\section{Conclusion}

To conclude, this study reported that the LP Nd: YAG laser is more effective in treating stretch marks than the fractional $\mathrm{CO}_{2}$ laser without serious adverse effects in spite of the insignificant difference between the two lasers histologically. The results of the present work concerning the adverse events of the two laser types encourage the use of the LP Nd: YAG laser in treating striae because the adverse effects were found to be higher with the fractional $\mathrm{CO}_{2}$ laser versus the LP Nd: YAG laser. It is recommended to perform other studies on a large scale of cases in order to support or oppose the conclusions of this research paper. Also, a long-term follow-up is required in future studies in order to have sufficient time for collagen fibers to be deposited in a significant amount.

\section{Ethical Considerations}

The approval of the research protocol was performed by the committee of research ethics, NRC, Giza, Egypt.

\section{Conflict of Interests}

The authors declare that they have no conflict of interest.

\section{References}

1. Shin JU, Roh MR, Rah DK, Ae NK, Suh H, Chung KY. The effect of succinylated atelocollagen and ablative fractional resurfacing laser on striae distensae. J Dermatolog Treat. 2011;22(2):113-21. doi: 10.3109/09546630903476902.

2. Youssef SES, El-Khateeb EA, Aly DG, Moussa MH. Striae distensae: Immunohistochemical assessment of hormone receptors in multigravida and nulligravida. J Cosmet Dermatol. 2017;16(2):279-286. doi: 10.1111/jocd.12337.

3. Brazil JC, Quiros M, Nusrat A, Parkos CA. Innate immune cell-epithelial crosstalk during wound repair. J Clin Invest. 2019;129(8):2983-2993. doi: 10.1172/JCI124618.

4. Cho S, Park ES, Lee DH, Li K, Chung JH. Clinical features and risk factors for striae distensae in Korean adolescents. J Eur Acad Dermatol Venereol 2006;20:1108-1113. doi: 10.1111/j.1468-3083.2006.01747. x.

5. Kim BJ, Lee DH, Kim MN, Song KY, Cho WI, Lee CK, et al. Fractional photothermolysis for the treatment of striae distensae in Asian skin. Am J Clin Dermatol. 2008;9(1):337. doi: 10.2165/00128071-200809010-00003.

6. Wanitphakdeedecha R, Meeprathom W, Manuskiatti W. A pilot study of treatment of striae distensae with variable square pulse Erbium: YAG laser resurfacing. $J$ Cosmet Dermatol. 2017;16(4):466-470. doi: 10.1111/jocd.12391.

7. Bretas T, Tanus A, Linhares M, Issa M.C. $\mathrm{CO}_{2}$ Laser for Scars. In: Issa $\mathrm{M}$, Tamura $\mathrm{B}$, eds. Lasers, Lights and Other Technologies. Clinical Approaches and Procedures in Cosmetic Dermatology. Springer; 2017:114. doi:10.1007/978-3-319-20251-8_13-1.

8. Abdel Hay R, Shalaby K, Zaher H, Hafez V, Chi C, Dimitri S, et 
al. Interventions for acne scars. Cochrane Database Syst Rev. 2016;(4):CD011946. doi: 10.1002/14651858.CD011946.pub2

9. Eichenfield DZ, Ortiz AE. Efficacy and Safety of the 532nm KTP and Long-Pulsed 1064-nm Neodymium-doped Yttrium Aluminum Garnet Laser for Treatment of Vascular Malformations. Dermatol Surg. 2020;46(12):1535-1539. doi: 10.1097/DSS.0000000000002386.

10. Wollina U, Goldman A. Management of stretch marks (with a focus on striae rubrae). J Cutan Aesthet Surg. 2017;10(3):124-129. doi:10.4103/JCAS.JCAS_118_17

11. Elsaie ML, Hussein MS, Tawfik AA, Emam HM, Badawi MA, Fawzy MM, et al. Comparison of the effectiveness of two fluences using long-pulsed Nd:YAG laser in the treatment of striae distensae. Histological and morphometric evaluation. Lasers Med Sci. 2016;31(9):18451853. doi: 10.1007/s10103-016-2060-2. Epub 2016 Sep 5.

12. Yang YJ, Lee GY. Treatment of Striae Distensae with Nonablative Fractional Laser versus Ablative CO2 Fractional Laser: A Randomized Controlled Trial. Ann Dermatol. 2011;23(4):481-9. doi: 10.5021/ad.2011.23.4.481.

13. Lee SE, Kim JH, Lee SJ, Lee JE, Kang JM, Kim YK, et al. Treatment of striae distensae using an ablative 10,600-nm carbon dioxide fractional laser: a retrospective review of 27 participants. Dermatol Surg. 2010;36(11):1683-90. doi: 10.1111/j.1524-4725.2010.01719. x.

14. Cho SB, Lee SJ, Lee JE, Kang JM, Kim YK, Oh SH. Treatment of striae alba using the 10,600-nm carbon dioxide fractional laser. J Cosmet Laser Ther. 2010;12(3):118-9. doi: 10.4103/0019-5154.177774.

15. Zaleski-Larsen LA, Jones IT, Guiha I, Wu DC, Goldman MP. A Comparison Study of the Nonablative Fractional 1565nm Er: glass and the Picosecond Fractional 1064/532-nm Nd: YAG Lasers in the Treatment of Striae Alba: A Split Body Double-Blinded Trial. Dermatol Surg. 2018;44(10):13111316. doi: 10.1097/dss.0000000000001555.

16. Güngör S, Sayilgan T, Gökdemir G, Ozcan D. Evaluation of an ablative and non-ablative laser procedure in the treatment of striae distensae. Indian J Dermatol Venereol Leprol. 2014;80(5):409-12. doi: 10.4103/0378-6323.140296.

17. Aust MC, Fernandes D, Kolokythas P, Kaplan HM, Vogt PM. Percutaneous collagen induction therapy: an alternative treatment for scars, wrinkles, and skin laxity. Plast Reconstr Surg. 2008;121(4):1421-1429. doi: 10.1097/01.prs.0000304612.72899.02.

18. Elsaie ML, Baumann LS, Elsaaiee LT. Striae distensae (stretch marks) and different modalities of therapy: an update. Dermatol Surg. 2009;35(4):563-73. doi: 10.1111/j.1524-4725.2009.01094.x.

19. Kravvas G, Veitch D, Al-Niaimi F. The use of energy devices in the treatment of striae: a systematic literature review. J Dermatolog Treat. 2019;30(3):294-302. doi: 10.1080/09546634.2018.1506078. 